University of Nebraska - Lincoln

DigitalCommons@University of Nebraska - Lincoln

$9-1-2002$

\title{
Nanoscale observation of photo-induced domain pinning and investigation of imprint behavior in ferroelectric thin films
}

\author{
Alexei Gruverman \\ University of Nebraska-Lincoln, agruverman2@unl.edu \\ B. J. Rodriguez \\ North Carolina State University, Raleigh, brian.rodriguez@ucd.ie \\ R. J. Nemanich \\ North Carolina State University, Raleigh
}

A. I. Kingon

North Carolina State University, angus_kingon@brown.edu

Follow this and additional works at: https://digitalcommons.unl.edu/physicsgruverman

Part of the Physics Commons

Gruverman, Alexei; Rodriguez, B. J.; Nemanich, R. J.; and Kingon, A. I., "Nanoscale observation of photoinduced domain pinning and investigation of imprint behavior in ferroelectric thin films" (2002). Alexei Gruverman Publications. 26.

https://digitalcommons.unl.edu/physicsgruverman/26

This Article is brought to you for free and open access by the Research Papers in Physics and Astronomy at DigitalCommons@University of Nebraska - Lincoln. It has been accepted for inclusion in Alexei Gruverman Publications by an authorized administrator of DigitalCommons@University of Nebraska - Lincoln. 


\title{
Nanoscale observation of photoinduced domain pinning and investigation of imprint behavior in ferroelectric thin films
}

\author{
A. Gruverman, ${ }^{\text {a) }}$ B. J. Rodriguez, R. J. Nemanich, and A. I. Kingon \\ Department of Materials Science and Engineering and Department of Physics, North Carolina State \\ University, Raleigh, North Carolina 27695-7920
}

(Received 18 March 2002; accepted for publication 6 June 2002)

\begin{abstract}
Piezoresponse force microscopy has been used to investigate the nanoscale mechanism of imprint behavior of ferroelectric $\mathrm{PbTiO}_{3}$ thin films by studying the photoinduced changes in the hysteresis loops of individual grains. Illumination of the film with UV light resulted in a voltage shift opposite to that observed in ferroelectric thin film capacitors. This effect is attributed to the generation of an electric field within the surface dielectric layer as a result of the interaction between photoinduced charges and polarization charges. Application of a small nonswitching bias to the film with simultaneous UV illumination resulted in domain pinning in the grains where the polarization direction coincided with the direction of the applied field, in agreement with the proposed model. Domain pinning was also observed in grains with polydomain structure suggesting that charge entrapment at the existing domain boundaries in the bulk of the film contributes to the suppression of switchable polarization. However, a symmetric character of hysteresis loops observed in such grains implies that charge entrapment in the bulk of the film does not cause the voltage shift. It has been suggested that a thin high-dielectric interfacial layer can improve the imprint behavior of ferroelectric capacitors. ㅇ 2002 American Institute of Physics. [DOI: 10.1063/1.1497698]
\end{abstract}

\section{INTRODUCTION}

Ferroelectric thin films are currently attracting significant interest due to the recent achievements in their integration into nonvolatile random access memories, ${ }^{1}$ which combine high-speed access, nearly unlimited endurance, and high radiation rigidity. One of the major limitations of the application of ferroelectric thin films in memory devices is the concern for long-term reliability characteristics of ferroelectric capacitors, which may be affected by various degradation effects. One such degradation effect is imprint, generally defined as a tendency of one polarization state to become more stable than the opposite one. ${ }^{2,3}$ This effect, which manifests itself by a shift of the polarization hysteresis loop along the voltage axis, can lead to the failure of a ferroelectric capacitor as a memory cell during the write operation since the capacitor cannot be switched by the programing voltage due to the increase in the coercive voltage at a certain polarization state. In addition, imprint leads to preferential suppression of switchable polarization. It is commonly accepted that imprint is caused by an asymmetric distribution of the trapped charge which screens the polarization state.

Imprint behavior in ferroelectric thin film capacitors has been the subject of numerous studies by conventional macroscopic techniques. ${ }^{4-11}$ Investigation of the photoinduced effects in ferroelectrics has proved to be a useful method for elucidating the mechanism of imprint. ${ }^{5,8-10}$ In addition, investigation of the interaction of the photogenerated carriers with the ferroelectric polarization can be useful from the viewpoint of the development of optical storage devices. However, there have been very few attempts to investigate

${ }^{a)}$ Electronic mail: alexei_gruverman@ncsu.edu the microscopic mechanism of imprint and to establish a correlation between voltage shift and domain switching. ${ }^{12}$ Recent progress in application of piezoresponse force microscopy (PFM) to high-resolution nondestructive characterization of ferroelectric thin films ${ }^{13-22}$ opens the way for direct investigation of the mechanism of imprint behavior at the submicrometer level via hysteresis loop measurements and domain structure imaging in individual grains. In this study, we utilize PFM to investigate the photoinduced changes in the voltage shift of the local hysteresis loops and in nanoscale domain switching in the $\mathrm{PbTiO}_{3}$ (PTO) thin films.

\section{EXPERIMENT}

The details and advantages of the PFM imaging method used in this study have been described in detail elsewhere. ${ }^{13}$ A modulation (imaging) voltage, applied through a conductive tip, causes a film vibration with the same frequency due to the converse piezoelectric effect. In the contact mode regime this results in the vertical oscillation of the cantilever which is detected using a standard lock-in technique. The vibration amplitude is proportional to the $d_{33}$ piezoelectric coefficient and represents the piezoelectric strain induced in the film. The polarization direction can be determined by monitoring the phase of the oscillation signal (piezoresponse) with respect to the imaging voltage. In the PFM image, domains of opposite polarity appear as regions of different contrast. In addition, simultaneous visualization of the surface topography allows establishing a correlation between film morphology and domain features.

In this study, domain imaging was performed using a commercial force microscope Autoprobe M5 (Park Scientific Instruments). A conductive gold coated $\mathrm{Si}_{3} \mathrm{~N}_{4}$ cantilever with 


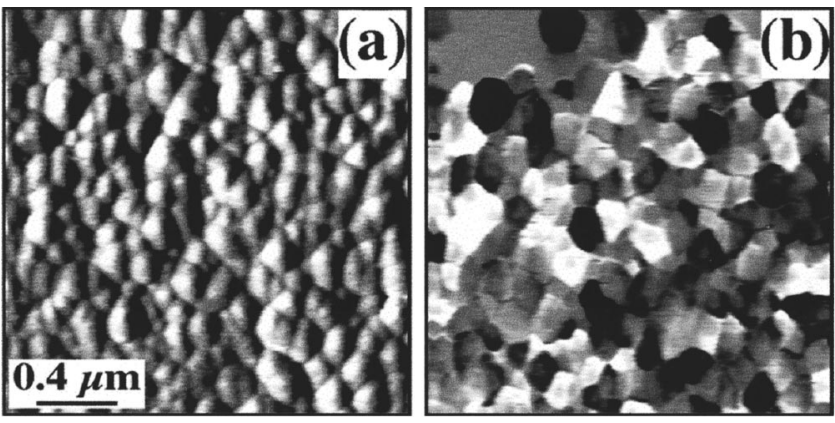

FIG. 1. Topographic (a) and piezoresponse (b) images of the PTO film. White and black regions in the piezoresponse image correspond to positive and negative domains, respectively.

a spring constant of $0.1 \mathrm{~N} / \mathrm{m}$ and a resonant frequency of 34 $\mathrm{kHz}$ was used both for domain visualization and for local hysteresis loop measurements. The domains were imaged by scanning the film with an oscillating tip bias of $1.5 \mathrm{~V}$ (peakto-peak) at $10 \mathrm{kHz}$. The hysteresis loop measurements were performed by positioning the probing tip at a selected grain and by measuring the PFM signal of the grain as a function of a dc voltage superimposed on the imaging voltage. The dc voltage was changed by a $0.25 \mathrm{~V}$ increment with a $2 \mathrm{~s}$ delay in the range from -3 to $3 \mathrm{~V} .{ }^{14}$ Hysteresis loops were measured before and after illumination of the sample with a band gap light (the primary energy at $254 \mathrm{~nm}$ ) using a $5 \mathrm{~W}$ PenRay Hg lamp. The imprint behavior was studied by measuring the hysteresis loops of grains with polarization along the $z$ axis, as detected in the PFM image. For the sake of clarity, phase and amplitude hysteresis loops were recorded separately.

Experiments were carried out using a 180-nm-thick PTO film deposited by a sol-gel technique onto a $\mathrm{Pt} / \mathrm{TiO}_{2} / \mathrm{Si}$ substrate. After deposition the film was annealed at $625^{\circ} \mathrm{C}$ for $30 \mathrm{~min}$. The x-ray diffraction analysis showed that the film exhibited preferential (001) and (110)/(101) texture.

\section{RESULTS AND DISCUSSION}

\section{A. Hysteresis and switching in as-grown PTO film}

A topographic image of the PTO film in Fig. 1(a) reveals a crystalline grain structure with lateral grain dimensions in the range from 80 to $240 \mathrm{~nm}$ and a median value of about $130 \mathrm{~nm}$. In the corresponding piezoresponse image [Fig. 1(b)], which contains information both on the amplitude and the phase of the PFM signal, grains with opposite out-ofplane polarization are represented by white and black regions. By monitoring the phase of the piezoresponse signal it was determined that the white regions in the PFM image correspond to domains with the polarization vector oriented toward the bottom electrode (hereafter referred to as "positive" domains), while the black regions correspond to domains oriented upward ("negative" domains). Histogram analysis of the PFM images showed symmetric distribution of positively and negatively polarized grains. In other words, the film did not show any significant macroscopic imprint. Based on our previous PFM analysis, ${ }^{13}$ it was concluded that grains with gray tone are randomly oriented so that their

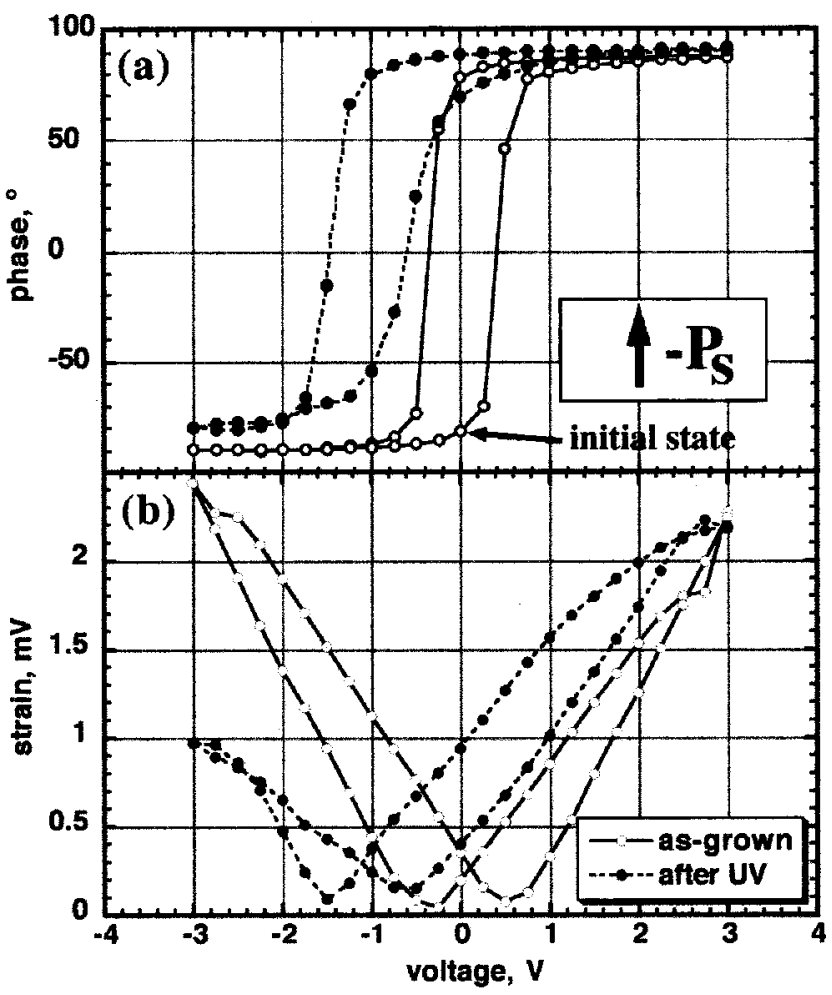

FIG. 2. Phase (a) and amplitude (b) PFM loops of a negatively polarized grain before and after UV illumination.

polarization vectors deviate from the film normal direction. A large region with zero piezoelectric activity in the upper left part of Fig. 1(b), which has quite different surface morphology, is most likely an amorphous phase that remains after annealing.

Figures 2 and 3 illustrate the initial hysteresis loops of the oppositely polarized grains. It can be seen that while the hysteresis loop of the negative grain is almost symmetric, the hysteresis loop obtained from the positive grain is shifted toward the negative bias. It should be noted that after the very first cycle of measurement the loops of the negative grains tend to shift toward the negative bias, while hysteresis loops in the positive grains did not show any change upon a number of measurements. Correspondingly, the switching behavior of the grains was strongly asymmetric. Application of a switching pulse to a negatively polarized grain resulted in complete inversion of the PFM contrast. In contrast, a positively polarized grain turned gray upon application of an opposite voltage pulse of the same amplitude. This behavior is similar to results obtained in ferroelectric lead zirconate titanate (PZT) films. ${ }^{22}$

It has been previously discussed that the voltage shift of the hysteresis loops in ferroelectric capacitors poled to a certain polarization state can be explained by the formation of space charges at the film/electrode interface. ${ }^{2,3}$ The polarization discontinuity near the film interface results in incomplete compensation of the depolarizing field by the charge on the electrodes. This leads to the existence of a residual depolarizing field, $E_{\mathrm{rd}}$, which is compensated via redistribution of electronic charges in the bulk of the film and subsequent formation of the space (screening) charge trapped near the film/electrode interface. ${ }^{23}$ The field of the space charge 


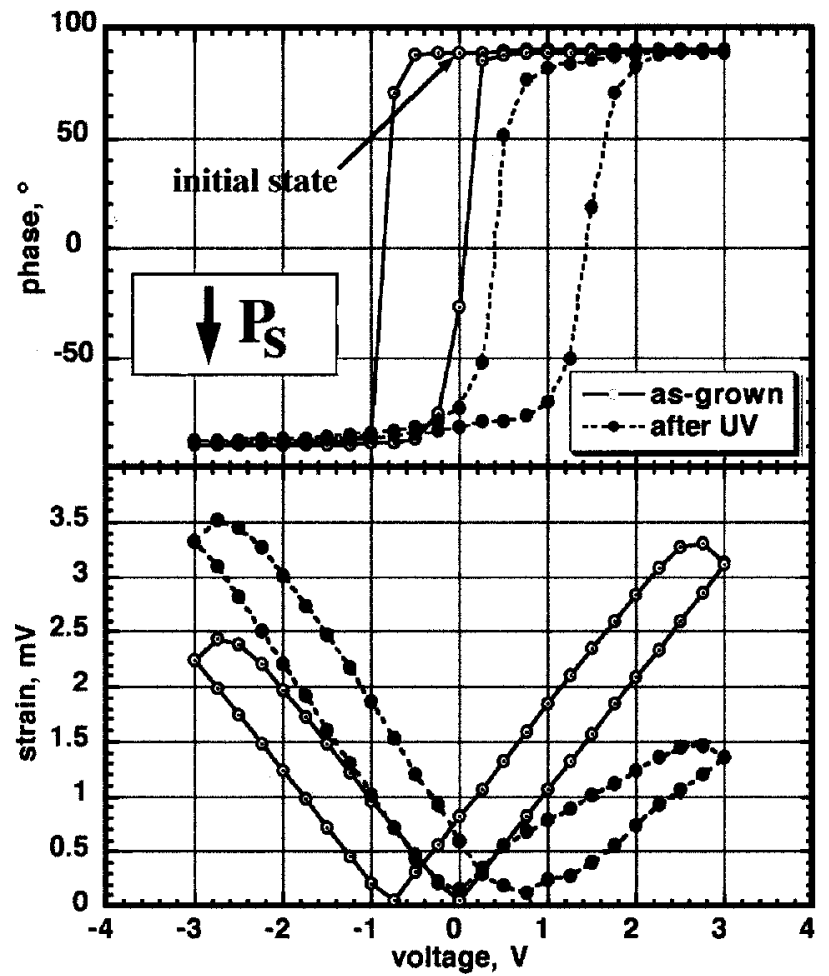

FIG. 3. Phase (a) and amplitude (b) PFM loops of a positively polarized grain before and after UV illumination.

causes a voltage shift of the hysteresis loops. Depending on the polarization state, this shift can be in the positive or negative direction: for example, the loop would be shifted toward positive voltages in a negatively polarized capacitor. ${ }^{2}$

However, the symmetric character of the PFM hysteresis loop in the negatively polarized grain suggests the presence of an additional source of an internal field, which is opposite to the screening field. Furthermore, the fact that the PFM loop observed in the positive grain is shifted implies that this field is polarization independent and points towards the bottom electrode. The presence of this built-in bias can be attributed to asymmetry of the boundary conditions at the top and bottom interfaces in the tip/film/electrode heterostructure. The presence of a Schottky barrier at the bottom interface has been recently suggested to be one of the reasons for asymmetric switching ${ }^{22}$ and the self-polarization effect in PZT films. ${ }^{24}$ It is thus possible that the effect of asymmetric switching described earlier is due to the inability of the electric field applied between the tip and the bottom electrode to reverse the positive polarization in the entire grain beneath the tip. This results in the formation of a "tail-to-tail" domain structure and leads to the gray contrast of the grain in the PFM image. ${ }^{22}$ This model also explains the suppressed strain response of the positive grain when a negative voltage is applied to the tip.

\section{B. Screening effects and photoinduced hysteresis changes}

To investigate the effect of the space charge on switching behavior, the hysteresis loop measurements were repeated after the sample was illuminated with UV light for 30
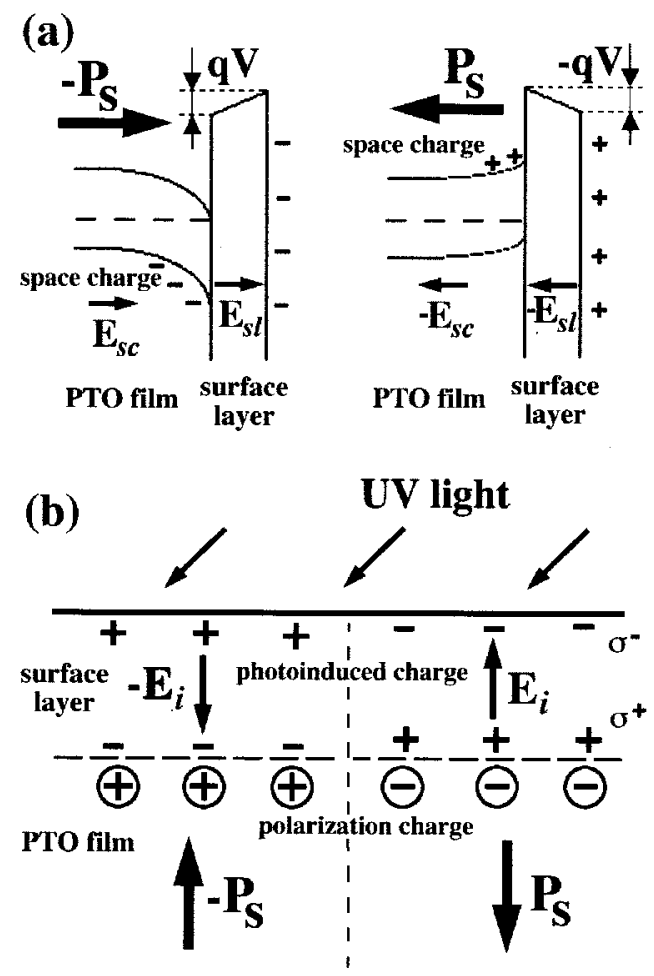

FIG. 4. A band diagram of a ferroelectric with the surface dielectric layer (a) and a schematic diagram (b) illustrating formation of the photogenerated field within the surface dielectric layer under UV illumination.

s. While UV illumination does not have any effect on the nanoscale domain pattern, it leads to significant changes in the voltage shifts of hysteresis loops with the sign of the shift determined by the polarization direction (Figs. 2 and 3). (It should be noted here, that the sample was not heated as a result of UV illumination.) In addition, UV illumination leads to an asymmetric suppression of the piezoelectric response. An example of this behavior is illustrated by the strain loops in Figs. 2(b) and 3(b). After UV illumination, relaxation of the photoinduced voltage shift has been observed which occurred within a time range of 20-50 min.

Let us emphasize that the UV-induced voltage shift observed in PFM is opposite to what was observed in $\mathrm{Pb}(\mathrm{Zr}, \mathrm{Ti}) \mathrm{O}_{3}$ and $(\mathrm{Pb}, \mathrm{La})(\mathrm{Zr}, \mathrm{Ti}) \mathrm{O}_{3}$ thin film capacitors. ${ }^{4,5}$ In those experiments, poling of a capacitor into the negative polarization state with simultaneous UV illumination led to a positive shift of the hysteresis loop, while in our experiments the loops of the negative grains are shifted to negative voltages under UV light. The same situation (with opposite sign) held for the positive polarization state. We suggest that this difference can be explained by taking into account the presence of a dielectric layer on the PTO film surface. A band diagram of a ferroelectric with the surface dielectric layer is shown in Fig. 4(a).

It has been reported previously that the as-grown PTO films have a surface dielectric layer (which is likely $\mathrm{PbO}$ that forms on film surface during the annealing process) with dielectric properties different from those of the bulk of the film. ${ }^{25,26}$ In the absence of the top electrode, the screening of polarization in the PTO film is provided both by the charge accumulated on the surface and the space charge formed at 
poling of as-grown film

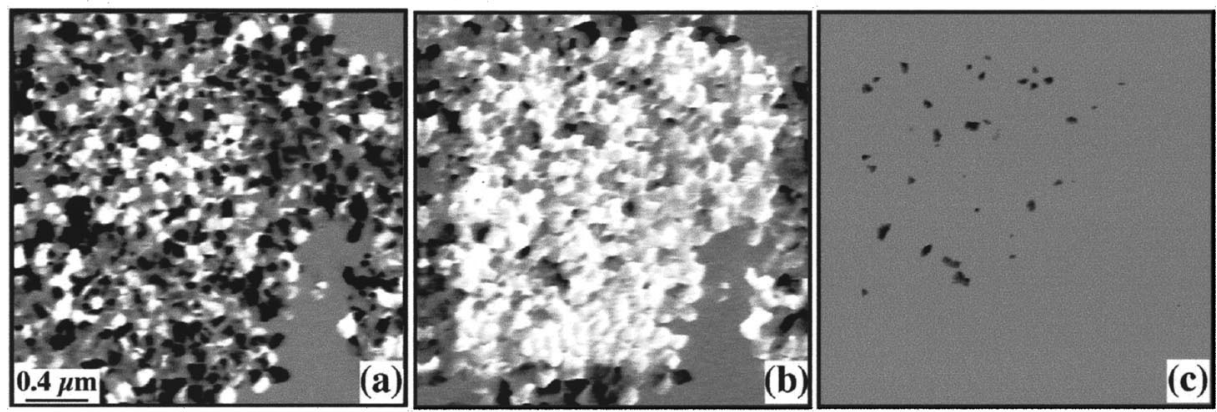

poling after $\mathrm{UV} / \mathrm{-1V}$ treatment
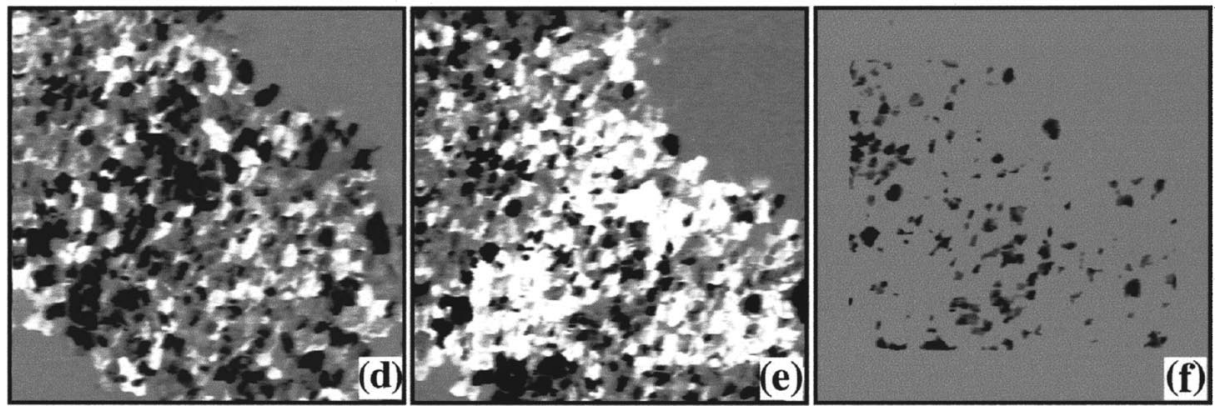

FIG. 5. Observation of domain pinning as a result of the UV/-1 V treatment. (a)-(c) Poling of as-grown PTO film, (d)-(f) poling after UV/-1 V treatment. (a), (d) PFM images of original domain structure; (b), (e) PFM images of domain structure after poling; (c), (f) differential PFM images obtained from the pairs of images (a), (b) and (d), (e), respectively. Dark areas in the differential PFM images depict regions where domain pinning occurred. Poling was performed by scanning the $2 \times 2 \mu \mathrm{m}^{2}$ area with a $+9 \mathrm{~V}$ dc bias applied to the tip. the vicinity of the ferroelectric-dielectric interface [Fig. $4(\mathrm{a})$. In a ferroelectric film with a dielectric layer of thickness $\ell$ and dielectric constant $\varepsilon_{\ell}$, the polarization screening by the surface charge leads to the formation of an electric field within this layer, which can be written as

$$
E_{s \ell}=\frac{P_{s}}{\varepsilon_{0}} \frac{d}{\left(\varepsilon_{\ell} d+\varepsilon_{d} \ell\right)},
$$

where $d$ is the ferroelectric film thickness, $\varepsilon_{d}$ is its dielectric constant, and $P_{s}$ is spontaneous polarization. The residual depolarizing field in the film interior is compensated by the field of the space charges

$$
E_{\mathrm{sc}}=\frac{P_{s}}{\varepsilon_{0}} \frac{\ell}{\left(\varepsilon_{\ell} d+\varepsilon_{d} \ell\right)} .
$$

The resulting voltage shift due to the screening charges, therefore, can be calculated as $V_{\text {shift }}=2 E_{s \ell} \ell+E_{\mathrm{sc}} d$.

Under UV illumination, the electrons and holes generated in the dielectric layer (we note here that $\mathrm{PbO}$ is known for its high photoconductivity in a wide range of wavelengths $)^{27}$ are separated due to their interaction with the polarization charges, e.g., in the negatively polarized grains electrons are attracted toward the ferroelectric-dielectric interface, while holes are repelled from it toward the surface [Fig. 4(b)]. A layer of the photogenerated charge of planar density $\sigma$ at the dielectric/ferroelectric interface will change the electric field within the dielectric layer by creating a photoinduced field $E_{i}$ [Fig. 4(b)]:

$$
E_{i}=\frac{\sigma}{\varepsilon_{0}} \frac{d}{\left(\varepsilon_{\ell} d+\varepsilon_{d} \ell\right)} .
$$

Obviously, the direction of the $E_{i}$ field depends on the grain polarity and is opposite to the $E_{s \ell}$ field. For a negatively polarized grain the field in the dielectric layer under
UV illumination can be written as $E_{s \ell}^{\prime}=E_{s \ell}-E_{i}$ which explains the shift of the hysteresis loop toward negative voltages. In the same way can be explained a voltage shift toward positive voltages in a positively polarized grain. The entrapment of the photoinduced charges stabilizes the existing domain configuration and suppresses its switching.

We suggest that the effect of carriers generated in the bulk of the film is less significant due to two reasons. First, the low field strength in the film interior, which differs from the field in the dielectric layer by a factor of $l / d$, should increase the time response of the bulk of the film compared to that of the dielectric layer and, for the time frame of UV illumination used in our experiments, should result in a weaker effect. Second and more important, the long-range migration of the photocarriers in the film is likely to be suppressed due to their interaction with defects and domain boundaries. As a result, the photogenerated carriers, instead of accumulating at the film interfaces, will be trapped and distributed throughout the film thickness and will not contribute to the voltage shift.

\section{Photoinduced domain pinning}

If the proposed model were correct, then application of a small nonswitching bias to the film with simultaneous UV illumination would lead to even stronger imprint in the grains where the polarization direction coincides with the direction of the applied field. In other words, application of a negative bias to the film surface will result in the effective stabilization of the polarization in the negative grains since the photoinduced charges will be trapped by deep centers within the dielectric layer. On the contrary, according to the bulk screening model, ${ }^{4}$ under an applied nonswitching negative bias the photogenerated carriers in the film interior would redistribute in such a way that they would destabilize 

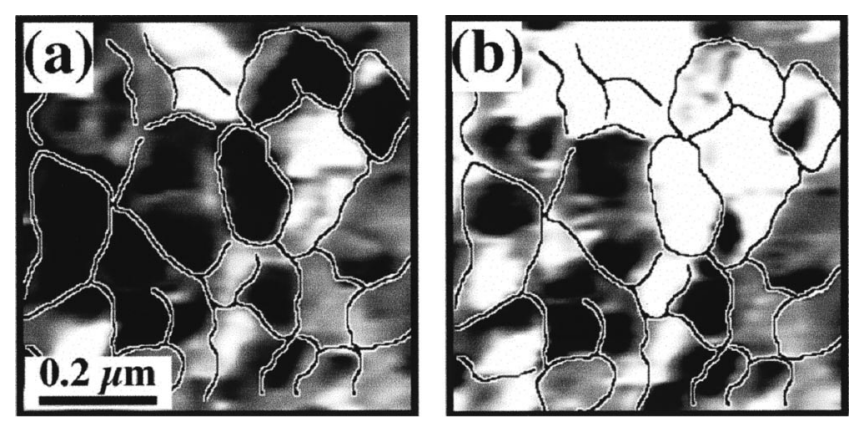

FIG. 6. PFM images of the film subjected to UV/-1 V treatment (a) before, and (b) after poling, illustrating domain pinning at the subgrain level. Black lines correspond to the grain boundaries.

the negative polarization and reduce imprint, since electrons would be forced to move towards the negative polarization end and holes towards the positive end. Indeed, it has been found that the negative polarization state has been further stabilized and negative imprint was enhanced by subjecting the film to a combined UV/-dc treatment.

The experiment was designed in the following way. First, to check the switchability of the as-grown film, a small area of the film $\left(2 \times 2 \mu \mathrm{m}^{2}\right)$ was poled by scanning with the tip under a dc bias of $+9 \mathrm{~V}$. PFM images of the film acquired before and after the dc voltage application [Figs. 5(a) and 5(b)] show that the film was effectively poled. Differential processing of the PFM images ${ }^{28}$ allowed us to extract an image of the unswitched grains that still maintain negative polarization after $+9 \mathrm{~V}$ poling [Fig. 5(c)]. The area fraction of these grains, normalized to the area originally with the negative polarization, is only about $4 \%$. To avoid an anomalous error due to the inhomogeneity of the film, the poling experiments were performed several times at different locations on the film surface. The results showed a reasonable degree of reproducibility with a standard deviation of about $20 \%$. The film was then subjected to a combined UV/-dc treatment, i.e., the film was illuminated with UV light while another area of $2 \times 2 \mu \mathrm{m}^{2}$ was scanned with the tip under a nonswitching (below coercive voltage) negative bias of -1 $\mathrm{V}$. Then this area was poled by applying a positive dc bias of $+9 \mathrm{~V}$ through the tip. The combined UV/-dc treatment of the film led to a significant increase in the area fraction where domain switching was suppressed due to domain pinning, as illustrated in Figs. 5(d)-5(f). The area fraction with pinned

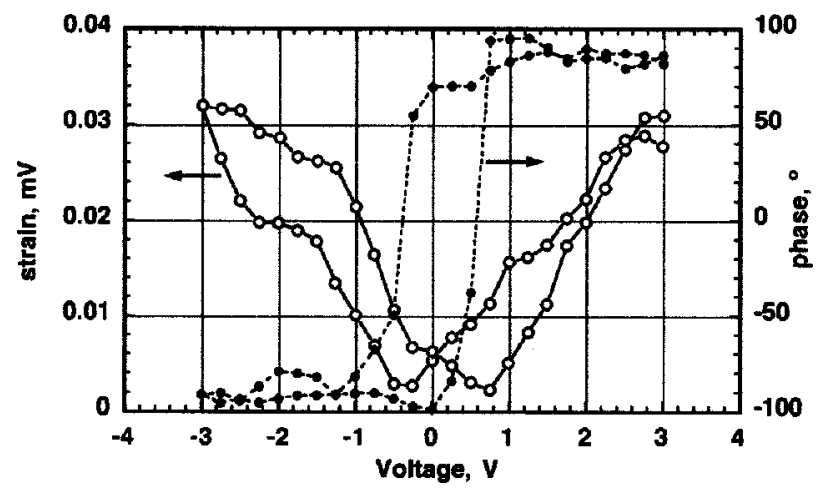

FIG. 7. Phase and amplitude PFM loops of a polydomain grain. negative domains comprised about $47 \%$ of the film area, which was in the negative polarization state before the $\mathrm{UV} /$-dc treatment.

It should be noted that examination of the pinned regions at higher magnification revealed that domain pinning is also pronounced in the grains which were originally in a polydomain state. [These grains appear with complex variations of black/white and gray contrast (Fig. 6).] At the same time, hysteresis loops measured in the polydomain grains did not show any voltage offset after UV illumination (Fig. 7) and exhibited weaker piezoresponse compared to the uniformly polarized grains. This result implies that the carriers generated in the film interior are trapped at the inner domain boundaries, which can lead to further suppression of the switchable polarization in agreement with the conclusion deduced from the observation of photoinduced changes in the fatigue behavior of $\mathrm{SrBi}_{2} \mathrm{Ta}_{2} \mathrm{O}_{9}$ and $\mathrm{Pb}(\mathrm{Zr}, \mathrm{Ti}) \mathrm{O}_{3}$ thin film capacitors. ${ }^{3,5,29}$ However, charge entrapment in the bulk of the film does not contribute to the voltage shift of the hysteresis loops.

The results presented here support the concept of a significant role of the electric field within the surface dielectric layer in the development of imprint. Obviously, parameters of the interface dielectric layers in ferroelectric capacitors are quite different from those in thin films which may account for the inverse photoinduced voltage shifts. The obtained results suggest that control of the dielectric layer properties provides an approach for improvement of imprint behavior of ferroelectric capacitors. It can be concluded from Eq. (1) that introducing a thin high-dielectric constant interfacial layer between capacitor electrodes and a ferroelectric layer will reduce the $E_{s l}$ field within the dielectric layer, and therefore, will lead to the reduced voltage shift in the ferroelectric capacitor.

\section{CONCLUSION}

In this study, piezoresponse force microscopy has been used to perform nanoscale measurements of the photoinduced changes in imprint behavior and in domain switching in lead titanate thin films. Observation of nanoscale domain pinning after combined UV/dc treatment provided direct experimental evidence of the mechanism of imprint behavior. It was shown that the voltage shift observed by PFM in individual grains of the PTO film under UV illumination is polarization dependent and is opposite to that observed in ferroelectric capacitors. This effect is explained by the formation of an electric field within the surface dielectric layer as a result of interaction between the photoinduced charges and the polarization charges. It is suggested, therefore, that the screening field that exists in the surface dielectric layer plays a significant role in imprint behavior of ferroelectric films by causing a voltage shift of the hysteresis loops and suppressing polarization switching. Charge entrapment at the existing domain boundaries in the bulk of the film also contributes to the suppression of switchable polarization but does not cause a voltage shift. It has been suggested that a thin highdielectric constant interfacial layer can improve the imprint behavior of ferroelectric capacitors. 
${ }^{1}$ O. Auciello, J. F. Scott, and R. Ramesh, Phys. Today 51, 22 (1998).

${ }^{2}$ W. L. Warren, D. Dimos, G. E. Pike, B. A. Tuttle, M. V. Raymond, R. Ramesh, and J. T. Evans, Appl. Phys. Lett. 67, 866 (1995).

${ }^{3}$ H. N. Al-Shareef, D. Dimos, W. L. Warren, and B. A. Tuttle, J. Appl. Phys. 80, 4573 (1996).

${ }^{4}$ D. Dimos, W. L. Warren, M. Sinclair, B. A. Tuttle, and R. W. Schwartz, J. Appl. Phys. 76, 4305 (1994).

${ }^{5}$ W. L. Warren, D. Dimos, B. A. Tuttle, R. D. Nasby, and G. E. Pike, Appl. Phys. Lett. 65, 1018 (1994).

${ }^{6}$ G. Arlt and H. Neumann, Ferroelectrics 87, 109 (1988).

${ }^{7}$ J. Lee and R. Ramesh, Appl. Phys. Lett. 68, 484 (1996).

${ }^{8}$ A. Kholkin, V. Yarmarkin, B. Goltsman, and J. Baptista, Integr. Ferroelectr. 35, 261 (2001).

${ }^{9}$ A. Kholkin, S. Iakovlev, and J. Baptista, Appl. Phys. Lett. 79, 2055 (2001).

${ }^{10}$ M. Grossmann, O. Lohse, D. Bolten, R. Waser, W. Hartner, G. Schindler, N. Nagel, and C. Dehm, Mater. Res. Soc. Symp. Proc. 541, 269 (1999).

${ }^{11}$ M. Grossmann, O. Lohse, D. Bolten, U. Boettger, R. Waser, W. Hartner, M. Kastner, and G. Schindler, Appl. Phys. Lett. 76, 363 (2000).

${ }^{12}$ M. Alexe, C. Harnagea, D. Hesse, and U. Gösele, Appl. Phys. Lett. 79, 242 (2001).

${ }^{13}$ A. Gruverman, O. Auciello, and H. Tokumoto, Annu. Rev. Mater. Sci. 28, 101 (1998)

${ }^{14}$ J. A. Christman, S.-H. Kim, H. Maiwa, J.-P. Maria, B. J. Rodriguez, A. I. Kingon, and R. J. Nemanich, J. Appl. Phys. 87, 8031 (2000).
${ }^{15}$ S. Hong et al., J. Appl. Phys. 89, 1377 (2001).

${ }^{16}$ T. Hidaka, T. Maruyama, I. Sakai, M. Saitoh, L. A. Wills, R. Hiskes, S. A. Dicarolis, and J. Amano, Integr. Ferroelectr. 17, 319 (1997).

${ }^{17}$ C. Harnagea, A. Pignolet, M. Alexe, and D. Hesse, Integr. Ferroelectr. 38, 23 (2001).

${ }^{18}$ H. Shin, J. Woo, S. Hong, J. Jeon, Y. E. Pak, and K. No, Integr. Ferroelectr. 31, 163 (1998).

${ }^{19}$ C. S. Ganpule, A. Stanishevsky, Q. Su, S. Aggarwal, J. Melngailis, E. Williams, and R. Ramesh, Appl. Phys. Lett. 75, 409 (1999).

${ }^{20}$ J. W. Hong, W. Jo, D. C. Kim, S. M. Cho, H. J. Nam, H. M. Lee, and J. U. Bu, Appl. Phys. Lett. 75, 3183 (1999).

${ }^{21}$ S. V. Kalinin and D. A. Bonnell, Phys. Rev. B 63, 125411 (2001).

${ }^{22}$ A. Gruverman, A. Kholkin, A. Kingon, and H. Tokumoto, Appl. Phys. Lett. 78, 2751 (2001).

${ }^{23}$ V. M. Fridkin, Photoferroelectrics (Springer, Berlin, 1979).

${ }^{24}$ A. Kholkin, K. Brooks, D. Taylor, S. Hiboux, and N. Setter, Integr. Ferroelectr. 22, 525 (1998).

${ }^{25}$ B.-D. Qu, Y.-G. Wang, W.-L. Zhong, K.-M. Wang, and Z.-L. Wang, J. Appl. Phys. 71, 3467 (1992).

${ }^{26}$ S. S. Dana, K. F. Etzold, and J. Clabes, J. Appl. Phys. 69, 4398 (1991).

${ }^{27}$ H. E. Brown, Lead Oxide: Properties and Applications (International Lead Zinc Research Organization, New York, 1985).

${ }^{28}$ A. Gruverman, Appl. Phys. Lett. 75, 1452 (1999).

${ }^{29}$ D. Dimos, H. N. Al-Shareef, W. L. Warren, and B. A. Tuttle, J. Appl. Phys. 80, 1682 (1996). 